\title{
Gastro Intestinal Bleeding Identification in Endoscopy Videos using Canny Edge Detection and Removal Algorithm
}

\author{
Bipin Dev S $\mathbf{S}^{1}$, Sarath $\mathbf{M}^{2}$, Kiran W $\mathrm{S}^{3}$, Jayaram $\mathbf{J}^{4}$ \\ ${ }^{1,2}$ PG Student, Department of ECE, Sivaji College of Engineering and Technology, Manivila, Kanyakumari Dist, TamilNadu, India \\ ${ }^{3,4}$ Assistant Professor, Department of ECE, Sivaji College of Engineering and Technology, Manivila, Kanyakumari Dist, TamilNadu, India
}

\begin{abstract}
Edoscopy videos are used for finding the bleeding regions. The edge regions are first detected and removed. Because the edge regions and the bleeding regions share same Hue value and the bleeding regions and the non -bleeding regions has same luminance, a canny edge detector is used to detect the edge regions in L channel. Canny edge detector is used because it can detect more edge pixels and preserve more bleeding pixels. Canny edge detector performs based on canny edge algorithm. The methodology in edge removal algorithm includes edge detection, edge dilation and edge masking. Thus the identification of the bleeding is carried out.
\end{abstract}

Keywords: Canny edge detector, Edge detection, Super-pixel segmentation, Edge dilation, Edge removal

\section{Introduction}

Gastro intestinal bleeding identification in endoscopy videos are used to determine the bleeding portions. In the case of modern technology over the medical fieid has an important role to overcome many critical situations. Wireless capule endoscopy technique is mainly used for identification of bleeding. The suspected blood indicator designed by Given Imaging Ltd. can only detect the active bleeding regions in small intestine. Also, this software is known to have insufficient sensitivity and specificity. The performance is measured in terms of three parameters such as sensitivity, specificity and accuracy. High sensitivity means high capability of detecting bleeding frames. High specificity means high capability of avoiding false detection. Accuracy is used to evaluate the overall performance. Since CAD is used to screen for bleeding WCE frames and send them to clinicians for specific examination, sensitivity is more important than specificity and accuracy The Wireless Capsule Endoscopy technique is done using a wireless capsule. The capsule endoscope provides a simple, safe, non-invasive, reliable procedure, well accepted and tolerated by the patient, which avoids any sedation, surgery or radiation exposure. Edge Detection scheme is useful to find out exact region of bleeding. With the support of edge dilation and edge removal all the regions are seperated from the bleeding locations. If the bleeding is critical then it is useful for identifying faster and easily. Take medical depends upon the circumstances.

\section{Related Works}

Edges are detected using different detectors. Before that there are different computational steps, such as the detection and localization criteria for a class of edge by considering signal to noise ratio, root mean square distance and present some mathematical forms for these criteria as functional on the operator impulse response and thus eliminating multiple responses[1]. The WCE images are diagnosed using computerized methods. Here color texture feature is used to judge the status of gastrointestinal tract. A new idea of chromaticity moment as the color feature, which make full use of the Tchebichef polynomials and the illumination invariant of the HSI color space. The texture is represented through Local Binary Pattern (LBP) which describes the spatial structure of local image texture[3].Training samples are rebalanced by over-sampling the minority classes and under-sampling the majority class. It re-samples the training data set such that balances of positive and negative samples are restored[4]. WCE is the state of art technology to examine gastro-intestinal tract. Here a novel strategy to segment WCE video clips based on abnormality which is based on a non-parametric corner detection method. Here the Most Representative Frames are extracted using k-means clustering method. The most representative frames are extracted by taking the Mutual Information from the adjacent frames[7]. The multi-resolution property of WCE images are extracted using combining uniform Local Binary Pattern (LBP) and wavelet transform.. The image pixels in the neighbourhood are first compared to the central pixel value and then the difference between the central pixel and its neighbours are labelled using the indicator functions[8]. a new image processing methods using combination of local feature for ulcer detection. It is based on Bags-of-words model and feature fusion technique. Image patches are described by LBP and SIFT feature (Scale-Invariant Feature Transform) and extracted using support vector machines classifier[9].Support Vector Machines (SVM) classifier is the main engine to learn and classify the system. Here an equal amount of bleeding and normal areas are taken for analysis. The current tested image is compared with the group of reference sequence in the database and finding the group having largest similarity and uses them as training data[10]. Detecting the bleeding regions automatically using the expectation maximization (EM) clustering algorithm and Bayesian information criterion (BIC) has been proposed. The EM algorithm is an efficient iterative procedure to compute the maximum likelihood (ML) estimate in the presence of missing or hidden data. Each iteration of the EM algorithm consists of two processes: the E-step and M-step[15]. Online training, compared with offline training, offers the advantage of adjusting the neural network over and over by taking into 


\section{International Journal of Science and Research (IJSR) \\ ISSN (Online): 2319-7064 \\ Index Copernicus Value (2013): 6.14 | Impact Factor (2015): 6.391}

account previously acquired knowledge (memory). Online $\mathrm{BP}$ is widely used in online neural network training; however, here it suffers from excessive sensitivity to learning parameters [19].

\section{Methodology}

A new method that can detect bleeding regions from WCE video more effectively and efficiently. For this the concept of edge pixels are taken in account. Since edge pixels and bleeding pixels share similar hue, where hue represents the actual wavelength of color, here RED color. Also in edge regions red intensities of color are more just like the bleeding regions, due to the presence of blood. Traditional algorithms often mistake edge pixels for bleeding pixels. Also In a typical WCE image, the most distinguishable feature between edge pixels and other non-bleeding pixels is the luminance.

The bleeding pixels and non-bleeding pixels (except edge pixels) normally have similar luminance and differ in redness. Therefore the bleeding pixels can't be removed, so the edge regions are first detected and removed. The most distinguishable feature between edge pixels and other non-bleeding pixels in a typical WCE image is the luminance. So the images are first converted from RGB space to CIE lab space. Canny edge detector is used because it can detect more edge regions and can preserve more bleeding regions. Through edge masking the intensities of the weak edges pixels are assigned as zero and are removed. The most distinguishable feature between edge pixels and other non-bleeding pixels in a typical WCE image is the luminance. So the images are first converted from RGB space to CIE lab space. Canny edge detector is used because it can detect more edge regions.and can preserve more bleeding regions. Through edge masking the intensities of the weak edges pixels are assigned as zero and which are removed systematically. Using this approach, absolute area of bleeding can be identified easily.

\subsection{Proposed Block Diagram}

The proposed system includes edge removal. Before edge removing the images are pre-processed. The pre-processing steps include Frame Conversion, and De-noising of image. The figure 1 shows the proposed block diagram for edge detection.

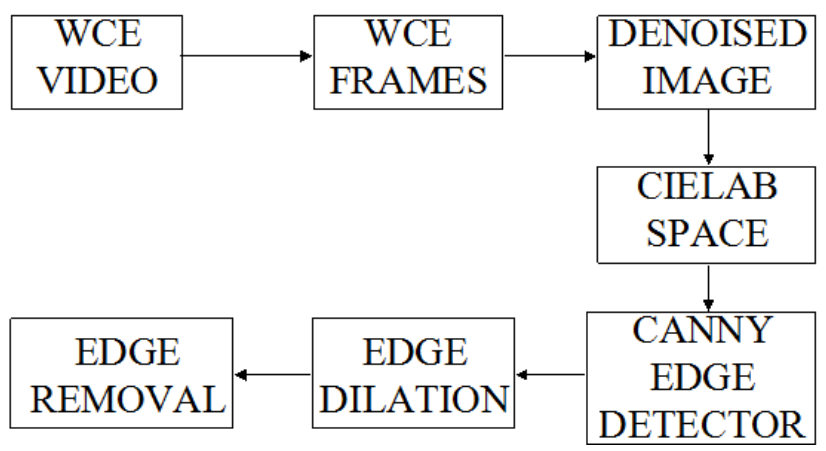

Figure 1: Proposed Block Diagram

In pre-processing video is converted as frames. The size of a film frame varies, depending on the still film format or the motion picture film format. A film frame or video frame is one of the many still images which compose the complete moving picture. The video will be read and convert the video as a frames. The converted frame will be saved. The process of elimination of uninformative regions (dark or bubbled regions) in a frame can make impact on detection of abnormalities.

The WCE images are often noisy. The noises are removed and the process is called de-noising. Noise reduction techniques are conceptually very similar, regardless of the image being processed. The median filter is a nonlinear digital filtering technique, often used to remove noise.

Before edge detection the WCE images are converted from RGB to CIELab space. In this color space we use three components: $\mathrm{L}$ is the luminance (brightness or intensity), a and $\mathrm{b}$ are respectively red/blue and yellow/blue chrominance.The color similarity between pixels can be measured in the CIE Lab spaceas uniform changes of components in CIE Lab correspond to uniform changes in perceived color.

\subsubsection{Canny Edge Detection Algorithm}

The edge pixels are detected using canny edge algorithm. The canny edge algorithm can be broken down into five steps.

1) Apply Gaussian filter to smooth the image in order to remove the noise

$$
H_{i j}=\frac{1}{2 \pi \sigma^{2}} \exp \left(-\frac{(i-k-1)^{2}+(j-k-1)^{2}}{2 \sigma^{2}}\right)
$$

2) Find the intensity gradients of the image

$$
\begin{array}{r}
G=\sqrt{\left(G x^{2}+G y^{2}\right)} \\
\Theta=a \tan 2(G y, G x)
\end{array}
$$

where Gx, Gy are the intensity gradient along horizontal and vertical direction.

3) Apply non-maximum suppression to get rid of spurious response to edge detection.

4) Apply double threshold to determine potential edges.

5) Track edge by hysteresis: Finalize the detection of edges by suppressing all the other edges that are weak and not connected to strong edges.

\subsubsection{Edge Dilation}

After edge detection, those regions are dilated using mathematical morphological method. For edge dilation two sets of input is needed, A is the image to be dilated. $\mathrm{B}$ is a set of coordinate points known as a structuring element (also known as a kernel) which determines the precise effect of the dilation on the input image.

$$
A \oplus B=\{z|B z \cap A|=\varnothing\}
$$

This equation is based on obtaining the reflection of B about its origin and shifting this reflection by $z$. The dilation of $A$ by $\mathrm{B}$ then is the set of all displacements, $\mathrm{z}$, such that B and A overlap by at least one pixel. Thus enlarged images of edges are obtained. 


\section{International Journal of Science and Research (IJSR) \\ ISSN (Online): 2319-7064 \\ Index Copernicus Value (2013): 6.14 | Impact Factor (2015): 6.391}

3.1.3. Edge Removal Algorithm

1) Convert the WCE image from RGB space to CIELab space. For the L Channel

2) Detect the edge regions using canny edge detector

3) Dilate the detected regions using mathematical morphological method

4) Locate the edge regions by masking, where the intensities of pixels are set to zeros

These are the important steps carried out for the edge removal or edge masking in the systematic manner. With the support of this result identification of the bleeding regions are carried out. The occurrence of errors are negligible under this condition due to three steps of process. Which can remove all the kinds of noises and non-bleeding parts. These steps are improving the accuracy as well as exact region of identification to get clear portion of affected area in the intestine.

\section{Experimental Results and Discussion}

The edge pixels or the regions of the wireless capsule endoscopy images are detected and removed using different process that are mentioned above. The results of those processes in WCE images are shown below:

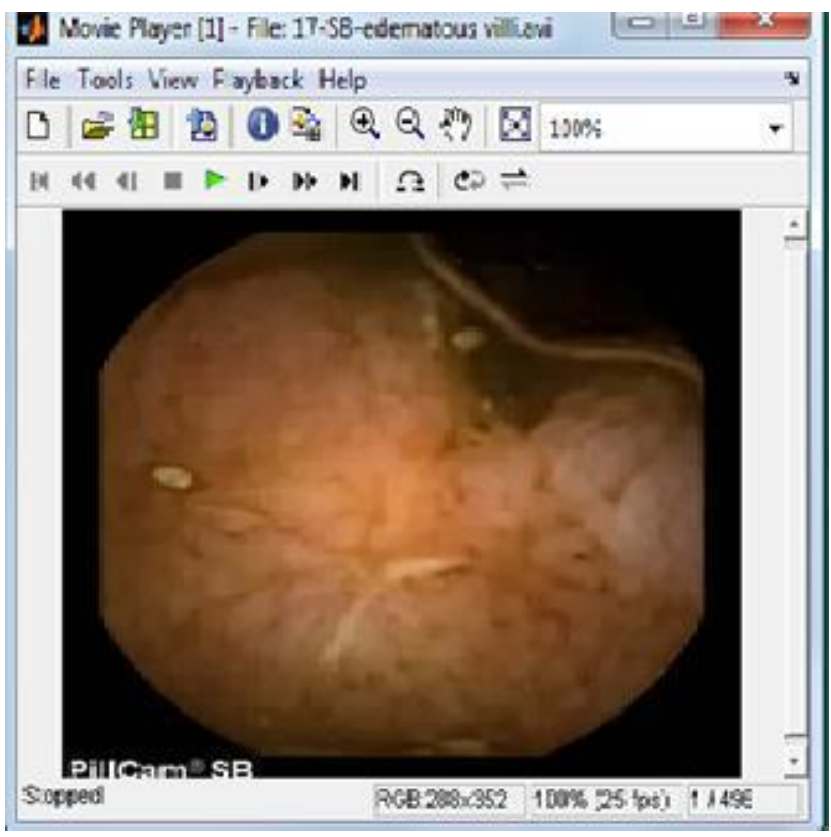

Figure 2: Video File Uploading

The video files are first uploaded from SBpillcam as in figure 2 . The videofile is in avi format. The dimensions are 288x352. From this video the corresponding bleeding areas are detected through the whole process.

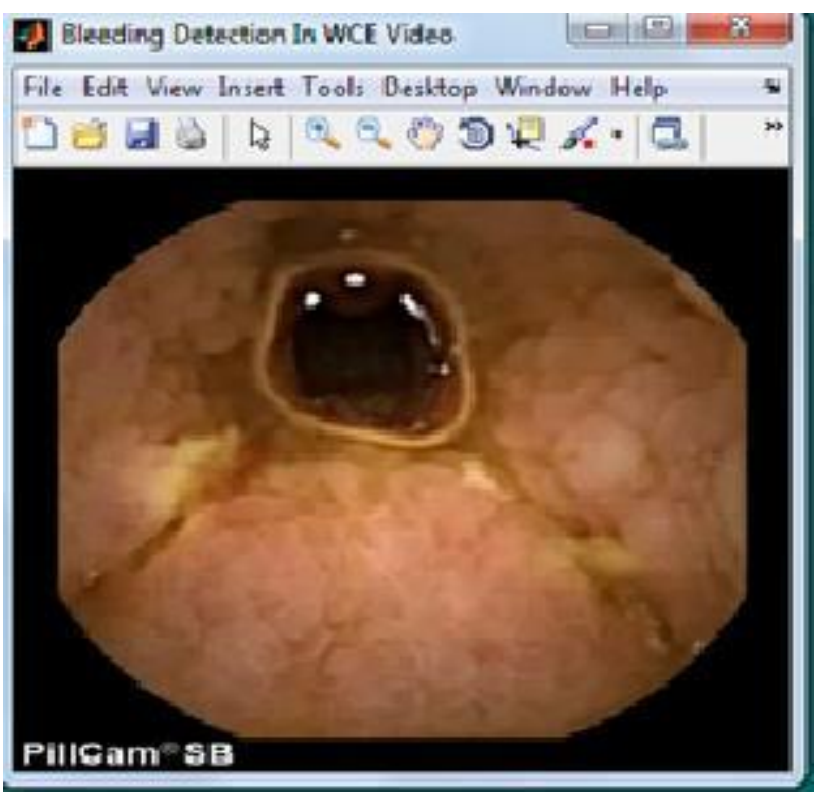

Figure 3: Frame Output

The video are first converted in to frames as in figure 3 . The video contain 496 frames. Each frame has 288x352 dimension. The frames are captured using SBpillcam.
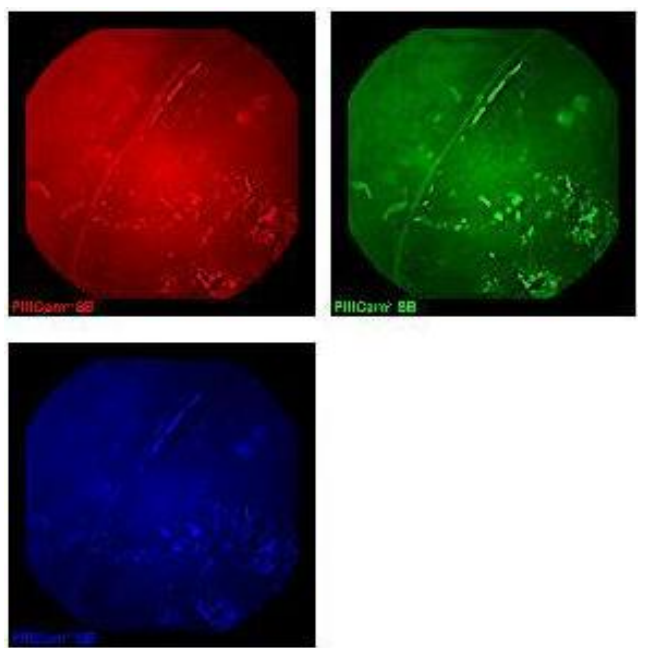

Figure 4: RGB Components

Before processing, the WCE frames are decomposed into three channels such as Red channel, Green channel and Blue channel. Which is highlighted in the figure 4.

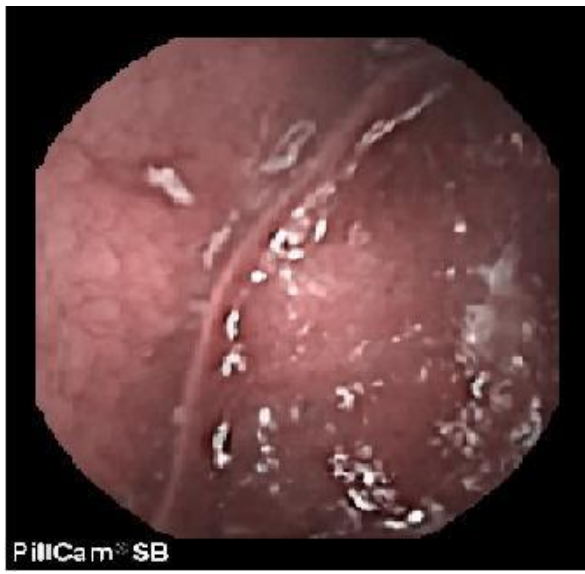

Figure 5: Denoised Image 


\section{International Journal of Science and Research (IJSR) \\ ISSN (Online): 2319-7064 \\ Index Copernicus Value (2013): 6.14 | Impact Factor (2015): 6.391}

The WCE images are often noisy. Therefore the noises are removed using median filter and Gaussian filter, thus a denoised image is obtained in RGB space.

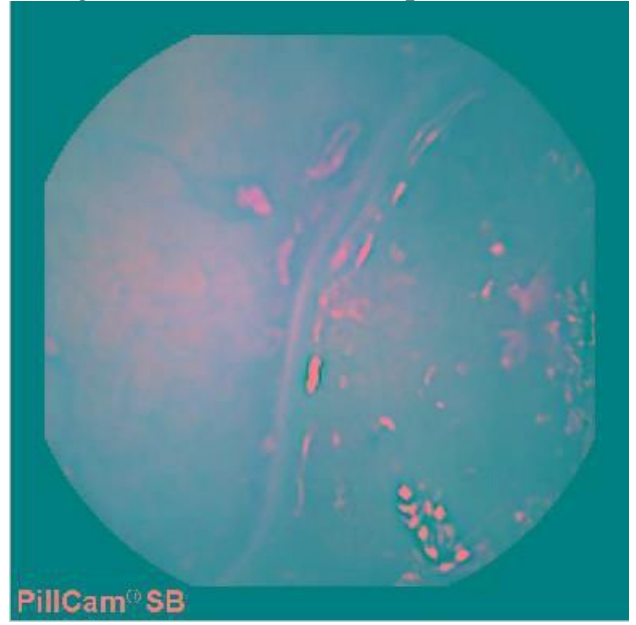

Figure 6: CIELab Image

Before edge detection the WCE images are converted from RGB to CIELab space. Since the luminance of the image are well defined in the CIELab space.

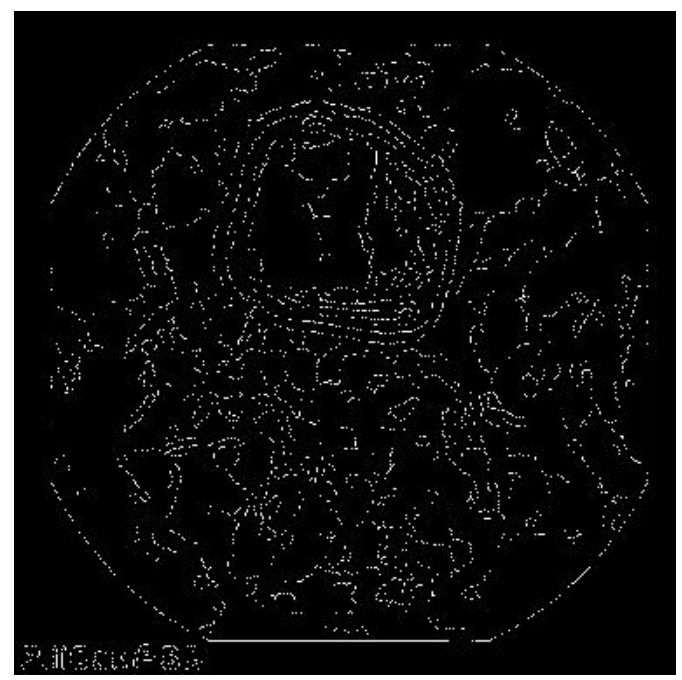

Figure 7: Canny Edge Image

The edge regions are detected using a canny edge detector. Canny edge detector is used as they can detect more edge regions and can preserve more bleeding regions as in figure 7.

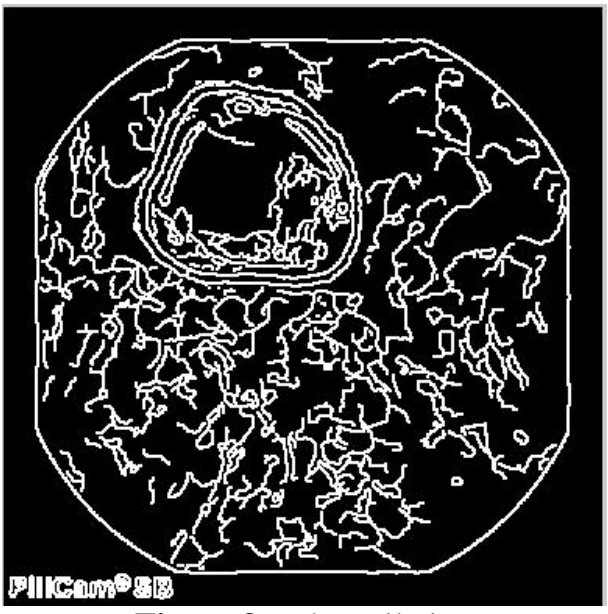

Figure 8: Edge Dilation
After edge detection those regions are dilated by mathematical morphological dilation method. In the figure 8 bleeding region identification is possible perfectly due to the dilation of canny edge image.

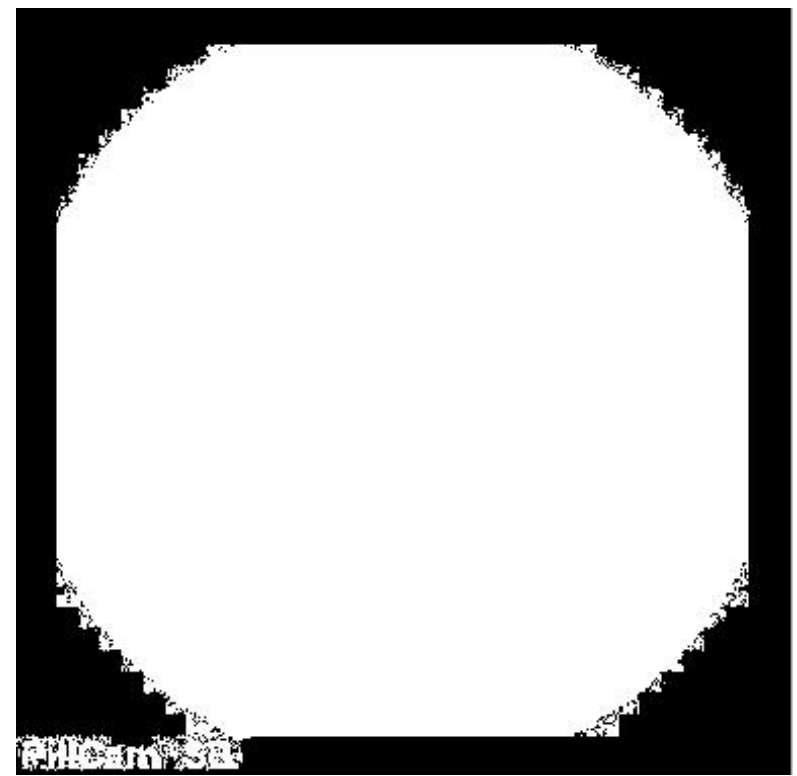

Figure 9: Edge Removal

The edge removed regions are shown above. The remaining regions are bleeding and non bleeding regions. Figure 9 is the indication of the whole process in the right way for the identification of edges. Thus the absolute classification is possible with these data sets of edges. Proper indications are provided by bleeding and non bleeding regions and it is stated as an absolute proof with the support of edge removal.

\section{Conclusion and Future Work}

The edge regions are detected and removed using canny edge detector, which can detect more edge regions so that more bleeding regions can be preserved than any other edge detection method. Thus well defined edge regions are detected and are removed through masking. In the future those edge removed regions are segmented through super-pixel segmentation and classified as bleeding and non bleeding regions using artificial neural network by using RBF algorithm and compares with other ANN algorithms and finally the performance are measured in terms of accuracy, specificity and sensitivity. The advanced classification is possible with neural network for identifying the minute bleeding regions.

\section{References}

[1] Alexandros Karargyris and Nikolaos Bourbakis,(2010)."A survey on various methodologies",IEEE Engineering in Medicine and Biology Magazine.

[2] Balathasan Giritharan, Xiaohui Yuan and Jianguo Liu,(2008)."Bleeding Detection from Capsule Endoscopy Videos", 30th Annual International IEEE EMBS Conference Vancouver, British Columbia, Canada.

[3] B. Li and M. Meng,(2009) "Computer-aided detection 


\section{International Journal of Science and Research (IJSR) ISSN (Online): 2319-7064 \\ Index Copernicus Value (2013): 6.14 | Impact Factor (2015): 6.391}

of bleeding regions for capsule endoscopy images" ,IEEE Trans. Biomed. Eng., vol. 56, no. 4,pp. 1032-1039.

[4] B. Penna, T. Tillo, M. Grangetto, E. Magli, and G. Olmo,(2009). "A technique for blood detection in wireless capsule endoscopy images",in Proc. 17th Eur. Signal Process. Conf., Glasgow, Scotland, 2009, pp. 1864-1868.

[5] B. Li and M. Meng,(2009). "Computer-aided detection of bleeding regions for capsule endoscopy images",IEEE Trans. Biomed. Eng., vol. 56, no. 4, pp. 1032-1039.

[6] B. Li, M. Meng, and J. Lau,(2011). “Computer-aided small bowel tumor detection for capsule endoscopy",Artif. Intell. Med., vol. 52, no. 1, pp. 1116.

[7] B. Li and M. Meng,(2012) "Tumor recognition in wireless capsule endoscopy images using textural features and SVM-based feature selection”, IEEE Trans. Inform. Technol. Biomed., vol. 16, no. 3, pp. 323-329.

[8] B. Li and M. Q.-H. Meng,(2012). "Ulcer detection in capsule endoscopy images", Imag. Vis. Comput., vol. 27, no. 9, pp. 1336-1342.

[9] J. Canny.( 1986.). "A computational approach to edge detection", IEEE Trans. Patt. Anal. Mach. Intell., vol. 8, no. 6, pp. 679-698,

[10] Jie Li, Jinwen Ma and Tamman Tillo,(2012)."A Training Based Support Vector Machine Technique For Blood Detection In Wireless Capsule Endoscopy Images", IEEE EMBS International Conference on Biomedical Engineering.

[11] M. Liedlgruber and A. Uhl,(2011). "Computer-aided decision support systems for endoscopy in the gastrointestinal tract: A review", IEEE Rev. Biomed. Eng., vol. 4, pp. 73-88.

[12] Miguel Tavares Coimbra and Joao Paulo Silva Cunha,(2006)."MPEG-7VisualDescriptors-Contributio ns for Automated Feature Extraction in Capsule Endoscopy",IEEE Transactions on Circuits and System for Video Technology,Vol.16,No.5.

[13] Phooi Yee Lau, and Paulo Loato Correia,(2007). "Detection of bleeding patterns in wce video using multiple features", in Proc. IEEE 29th Annu. Int. Conf. Eng. Med. Biol. Soc., pp. 5601-5604.

[14] Poh Chee Khun, Zhang Zhuo, Liang Zi Yang, Li Liyuan, and Liu Jiang, (2009). "Feature Selection and Classification For Wireless Capsule Endoscopic Frames", Institute for Infocomm Research (I2R) Agency for Science, Technology and Research (A*STAR), IEEE.

[15] Qian Zhao, Max Q.-H. Meng and Baopu Li,(2010). "WCE Video Clips Segmentation based on Abnormality," 2010 IEEE International Conference on Robotics and Biomimetics, Tianjin, China.

[16] Tonmoy Ghosh and Khan Arif Wahid,(2014).”An Automatic Bleeding Detection in Wireless Capsule Endoscopy based on RGB pixel intensity ratio", International Conference on Electrical Engineering and Information \& Communication Technology (ICEEICT).

[17] T.Ghosh, S.K.Bashar,S.A Fattah,(2014)"An Automatic Bleeding Detection Scheme in Wireless Capsule Endoscopy based on Statistical Features in hue space",17th International Conference on Computer and Information Technology (ICCIT).

[18] V.S.Kodogiannis,M.boulougoura,(2005).’Neural network- based approach for the classification of wireless-Capsule endoscopic images", International Conference on Neural Network, IEEE.

[19] Y. Fu, M. Mandal, and G. Guo,(2011). "Bleeding region detection in wce images based on color features and neural network", in Proc. IEEE 54th Int. Midwest Symp. Circuits Syst., pp. 1-4

[20] Yixuan Yuan, Baopu Li, Max Q.-H. Men, (2015). "Bleeding Frame and Region Detection in the Wireless Capsule Endoscopy Video", IEEE, Journal of Biomedical and Health Informatics.

\section{Author Profile}

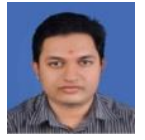

Bipin Dev S S received the B.E. Degree in Electronics and Communication Engineering from Marthandam College of Engineering and Technology in 2012. He was trained in Optical Communication from BSNL-RTTC in 2013. Now he is doing the M.E. Degree in Communication Systems from Sivaji College of Engineering and Technology.

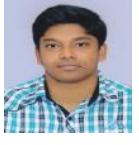

Sarath $\mathbf{M}$ received the B.E. Degree in Electronics and Communication Engineering from Muslim Association College of Engineering in 2014. Now he is doing the M.E. Degree in Communication Systems from Sivaji College of Engineering and Technology.

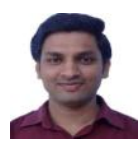

Kiran W S received the B.E. in Electronics and Communication Engineering from Vins Christian College of Engineering in 2011 and M.E. in Communication Systems from Karpagam College of Engineering in 2013. Now he is persuing Ph.D in Anna University. Currently, he is an assistant professor in Sivaji College of Engineering and Technology.

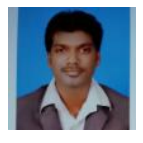

Jayaram $\mathbf{J}$ received the B.E. in Electronics and Communication Engineering from Sun College of Engineering and Technology in 2009 and M.E. in Computer and Communication from Rajas College of Engineering in 2012. Currently, he is an assistant professor in Sivaji College of Engineering and Technology. 